\title{
Simple method for confirming tibial osteotomy during total knee arthroplasty
}

\author{
Hirotaka Mutsuzaki ${ }^{1}$ and Kotaro Ikeda ${ }^{2^{*}}$
}

\begin{abstract}
Background: Achieving precise implant alignment is crucial for producing good outcomes after total knee arthroplasty (TKA). We introduce a simple method for confirming the accuracy of tibial osteotomy during TKA.

Findings: Two metallic markers were placed on the skin $20 \mathrm{~cm}$ apart, one on the tibial tuberosity and other on the tibial crest, points that are easily identified and palpated intraoperatively. Anteroposterior radiographs of the legs were obtained. We defined the line along the markers as the tuberosity line. The osteotomy line is perpendicular to the anatomical axis of the tibia. We then calculated the angle between these two lines and designated it the osteotomy angle. We set the osteotomy angle of the protractor, and cut the bone parallel to the osteotomy line of the protractor. Postoperatively, we analyzed the varus angle of the tibial osteotomy in 35 TKAs using the protractor. The average of the varus angle of the tibial osteotomy was $89.4^{\circ} \pm 1.6^{\circ}$ (95\% confidence interval of $\left.-1.0976,0.0119\right)$. There was no significant difference from the target angle of $90^{\circ}(p=0.055)$. The varus angles of $90^{\circ}$ and $90^{\circ} \pm 2^{\circ}$ for the tibial osteotomy were $42.9 \%$ and $82.9 \%$, respectively.
\end{abstract}

Conclusions: We determined the accuracy of the tibial osteotomy in the coronal plane using the protractor to be satisfactory.

Keywords: Total knee arthroplasty, Tibial osteotomy, Extramedullary, Alignment, Protractor

\section{Findings Introduction}

Correct alignment of the tibial and femoral components is one of the most important factors determining favorable long-term results of a total knee arthroplasty (TKA). It is generally accepted that the tibial component should be placed perpendicular to the anatomical axis of the tibia [1-3]. We use extramedullary guides for cutting the tibial bone because they help us avoid the potential complications of intramedullary guide use, including fat embolization, intraoperative fracture, and inability of intramedullary rod passage due to deformity [4]. Placement of the tibial component in excessive varus is the commonest cause of suboptimal alignment using extramedullary guides [5]. Coull et al. [6] showed that $48 \%$ of TKAs had tibial component angles of $<87^{\circ}$ even when they tried to cut the tibia perpendicular to its anatomical axis. Also, there was a significant decrease in the

\footnotetext{
* Correspondence: drikeda@mac.com

${ }^{2}$ Department of Orthopaedic Surgery, Ichihara Hospital, 3681 Ozone,

Tsukuba, Ibaraki 300-3295, Japan

Full list of author information is available at the end of the article
}

probability if the implant was inserted in any varus alignment [7]. Aglietti and Buzzi [8] demonstrated that varus alignment of the knee and a varus tilt of more than $2^{\circ}$ of the tibial component correlated with the incidence of lucent lines around the tibial implant. Computerassisted navigation, digital preoperative planning and patient-specific guides improved accuracy of tibial osteotomy in TKAs [9-12]. Recently, bone-cutting devices have improved accuracy of implant positioning, even if TKA is performed manually or using minimally-invasive technique $[13,14]$.

We developed our original intraoperative protractor to place on top of a tibial tuberosity and tibial crest so that we can easily palpate from the body's surface intraoperatively to confirm that the tibial osteotomy has obtained the desired tibial angle. Therefore, we hypothesized that it is possible to cut a bone precisely using a protractor intraoperatively. The purpose of this study was to evaluate the accuracy of the tibial bone cut angle using an intraoperative protractor during TKA.

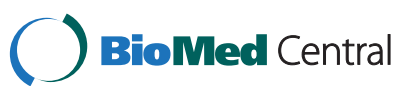




\section{Patients and methods}

From October 2000 to April 2002, a total of 3 men and 24 women (aged 23-87 years, mean 61.1 years) underwent 35 TKAs for osteoarthritis $(n=14)$ or rheumatoid arthritis $(n=21)$ using the Scorpio nonrestrictive geometry cruciate retaining system (Stryker Howmedica Osteonics; Allendale, NJ, USA). The ethics committee of Ichihara Hospital reviewed and approved the study (the reference number for the ethics approval: 1203, and the trial registration number: 1203). Informed consent was obtained from each patient. A single surgeon performed all of the operations. They were done with the patient under epidural or general anesthesia and using the medial parapatellar approach. The target alignment of tibia osteotomy was perpendicular to the anatomical axis of the tibia. We used our original intraoperative protractor and an extramedullary guide.

\section{Preoperative planning}

Two metallic markers were placed on the skin: one on the top of the tibial tuberosity and the other on the top of the anterior tibial crest (Figure 1a). The markers were $20 \mathrm{~cm}$ apart. These two points are easy to palpate from the surface of the body. Anteroposterior radiographs of the legs were obtained (Figure 1b). We constructed the angle of the osteotomy based on the X-ray films. We defined the line between the two markers as the tuberosity line. We defined the median line of the tibial shaft as the anatomical axis of the tibia in the coronal plane. The osteotomy line was perpendicular to the anatomical axis of the tibia. We then calculated the angle between the tuberosity line and anatomical axis of tibia. Finally, we calculated the angle of the osteotomy line with the tuberosity line and called it the osteotomy angle (Figure 1c).

\section{Intraoperative protractor method}

We made our original intraoperative protractor from stainless steel. The protractor is constructed with a $10-\mathrm{cm}$ bar and a $20-\mathrm{cm}$ bar. There is a point of intersection after one end is placed at the top of the tibial tuberosity and the distal $20 \mathrm{~cm}$ is at the top of the anterior tibial crest. Intraoperatively, we were able to confirm the two points easily. The angle made by the two bars is displayed on a scale at the intersection part (Figure 2). The 20-cm bar is in accord with the tuberosity line, and the $10-\mathrm{cm}$ bar is in synchrony with the osteotomy line. We set the osteotomy angle of the protractor based on a preoperative measurement. We marked a line parallel to the protractor as the proximal tibia by marker or

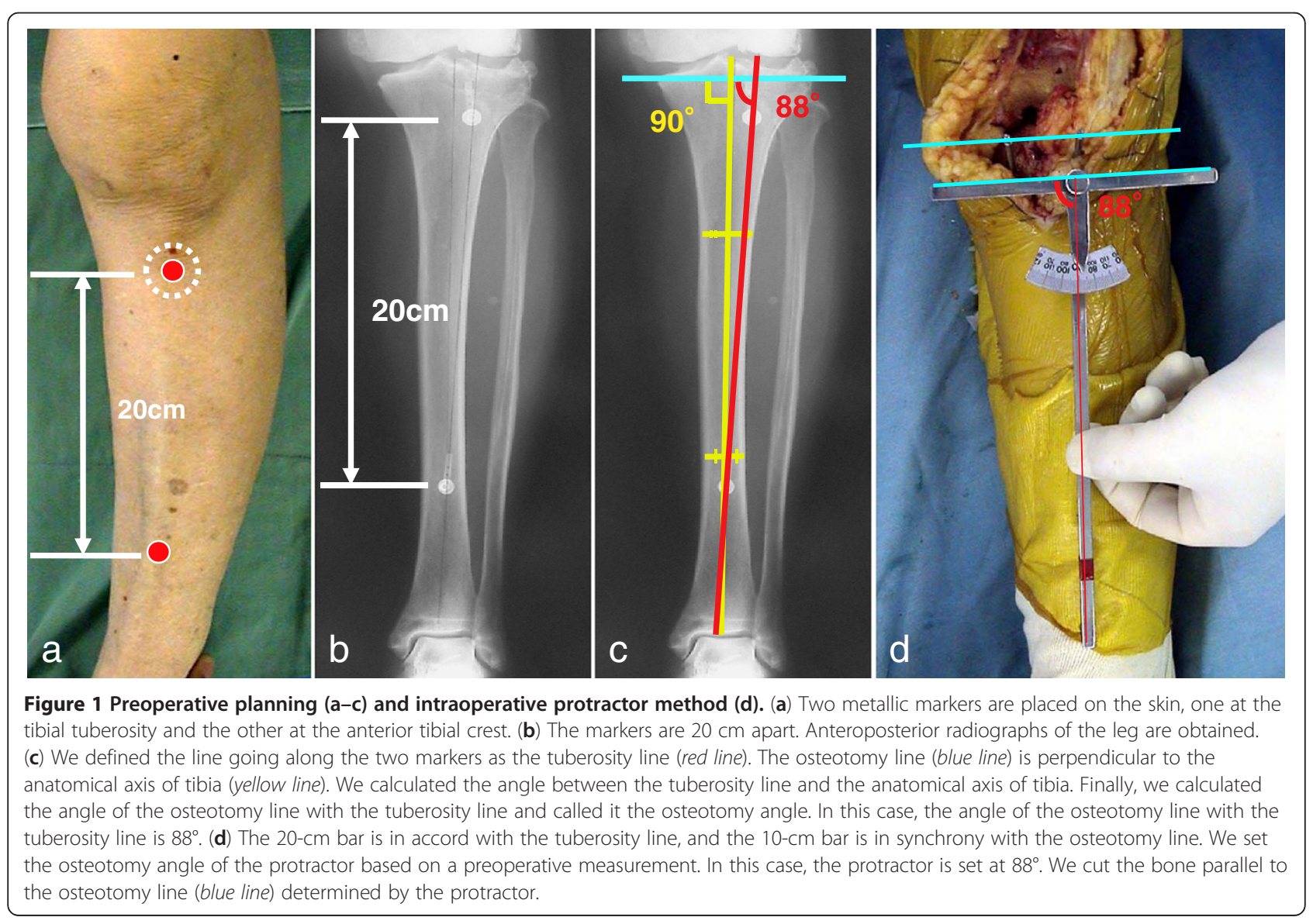



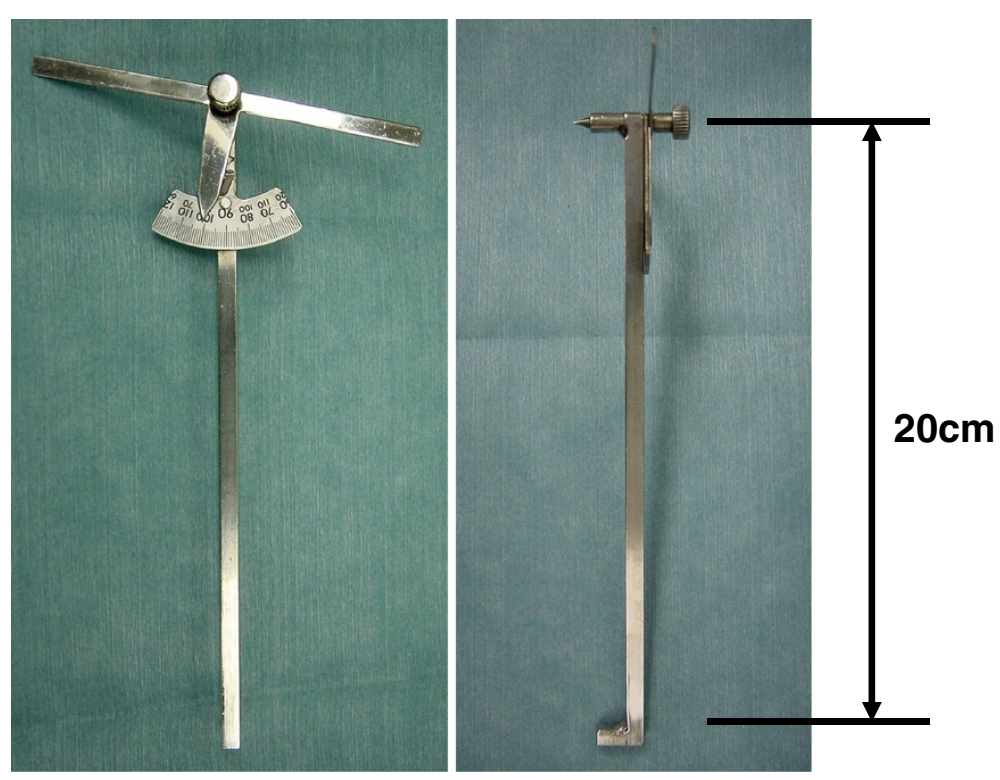

Figure 2 View of our original intraoperative protractor (right, anteroposterior view; left, lateral view). The protractor is constructed with a $10-\mathrm{cm}$ bar and a $20-\mathrm{cm}$ bar. There is a projection at a point of intersection where the $10-\mathrm{cm}$ bar is at the tibial tuberosity and the distal $20-\mathrm{cm}$ bar is at the anterior tibial crest. The angle made by the two bars make is displayed on a scale at their intersection.

electric knife. We adjusted the extramedullary cutting guide parallel to the mark. We then cut the bone parallel to the osteotomy line of the protractor with a posterior slope of $5^{\circ}$ in the sagittal plane (Figure 1d). The rotational alignment of the tibial component was adjusted to the anteroposterior axis between the center of the cut surface and the border of the medial third of the tibial tuberosity. Postoperatively, we analyzed the varus angle of the tibial osteotomy (Figure 3).

\section{Statistical analyses}

The data for the varus angle of the tibial osteotomy was compared with the target angle $\left(90^{\circ}\right)$ using Student's $t$ test, with $p<0.05$ indicating significance.

\section{Results}

The average varus angle of the tibial osteotomy using the protractor was $89.4^{\circ} \pm 1.6^{\circ}$ [95\% confidence interval (CI) was $-1.0976,0.0119]$. There was no significantly difference from $90^{\circ}(p=0.055)$. The $90^{\circ}$ and the $90^{\circ} \pm 2^{\circ}$ tibial osteotomy varus angles were $42.9 \%$ and $82.9 \%$, respectively.

\section{Discussion}

The average varus angle of the tibial osteotomies using the protractor was $89.4^{\circ} \pm 1.6^{\circ}(95 \%$ CI was -1.0976 , 0.0119). The intraoperative protractor method is simple, and the accuracy is high. The tibial tuberosity and the tibial crest are easily palpated, so it is easy to identify the tuberosity line during the operation. The plasticity of the intraoperative tuberosity line can be high. The protractor can be used to confirm the cutting line after setting osteotomy jigs as an indicator. Recently, the use of a computer-assisted navigation system that achieved a high degree of accuracy relative to the desired target alignment has been described $[9,10,14]$. However, unpredictable complications, such as displaced or stress femoral or tibial fractures, have been reported to occur a few weeks after the operation with the use of a computer navigation system $[15,16]$. The protractor method is simple and low-priced, and the accuracy is high.

There were no complications using the protractor method. However, there are a few cautions to be noted. Anteroposterior radiography of the tibia is necessary before the operation. Also, the tibial tuberosity must be well palpated to reproduce the position of the metallic marker before the surgery: $5 \mathrm{~mm}$ away from the tibial tuberosity can make the error of $1.5^{\circ}$ when performing the osteotomy. If there is deformity in the distal tibial crest where the marker has been placed, the tibial osteotomy may not be perpendicular to the tibial shaft. With these precautions, we found satisfactory accuracy for performing tibial osteotomy in the coronal plane using the protractor.

Regarding the limitation of this study, the present method was just 2-dimensional (2D), and accuracy of implant positioning was also performed with 2D radiographic analysis, which was far behind the current 3dimensional (3D) tools, such as computed tomography (CT)-based navigation $[9,10,14]$, 3D-preoperative planning software [11], and patient specific instrumentation system [12]. Therefore, a potential weakness of the study was the lack of spatial recognition. However, the present 


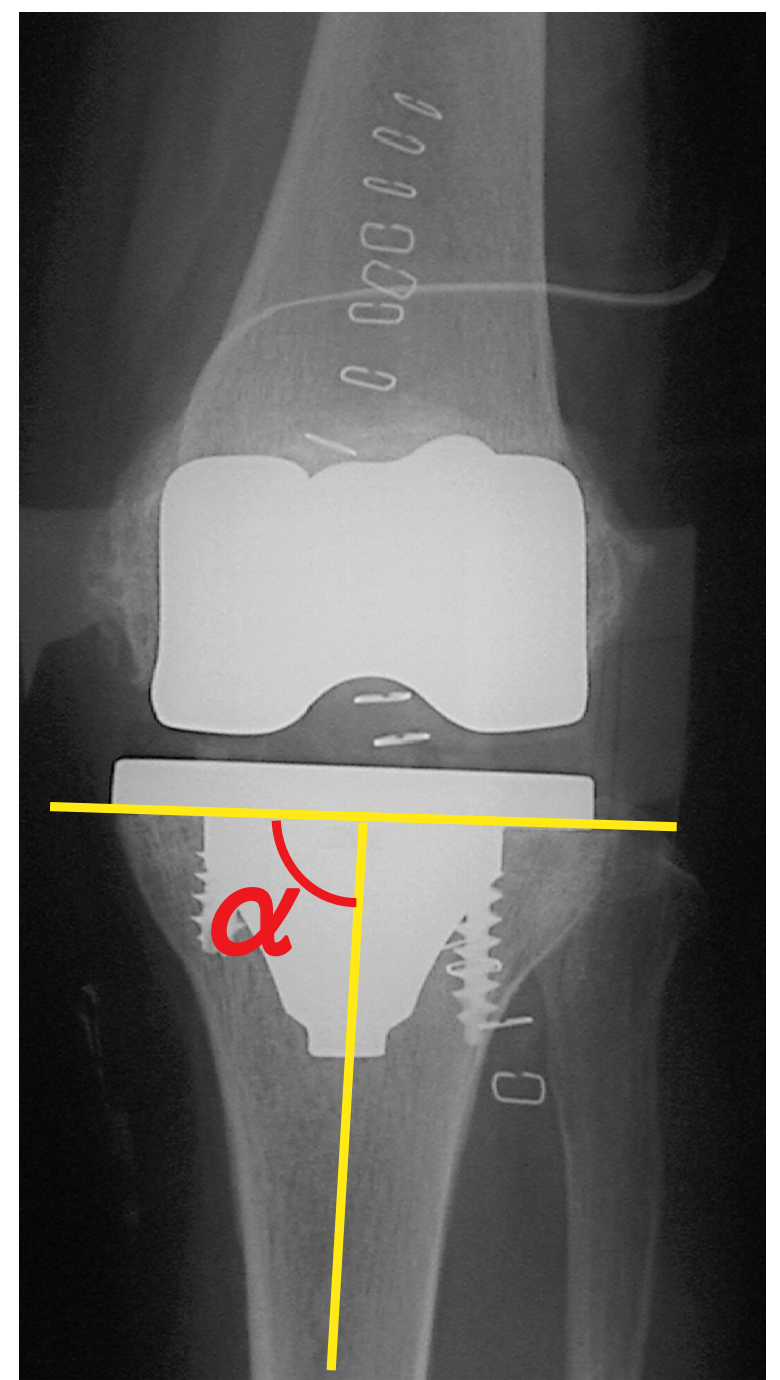

Figure 3 Anteroposterior radiograph of the knee. a: varus angle of the tibial osteotomy.

method without CT appears to have a merit in minimizing the risk of radiation exposure compared to the current tools. The study lacked a control group without the protractor, and we used small numbers of subjects. The comparative study between using the protractor and a conventional method using large numbers of subjects are necessary to clarify the effect of the protractor in the future.

\section{Competing interests}

Both authors declare that they have no competing interests.

\section{Authors' contributions}

$\mathrm{HM}$ and $\mathrm{KI}$ conceived of the study and participated in its design and coordination. HM participated in the sequence alignment and drafted the manuscript. All authors read and approved the final manuscript.

\section{Acknowledgments}

The authors thank Iso Medical Systems Inc., Tokyo, Japan for technical support.

\section{Author details}

Department of Orthopaedic Surgery, Ibaraki Prefectural University of Health Sciences, 4669-2 Ami Ami-machi, Inashiki-gun, Ibaraki 300-0394, Japan. ${ }^{2}$ Department of Orthopaedic Surgery, Ichihara Hospital, 3681 Ozone,

Tsukuba, Ibaraki 300-3295, Japan.

Received: 17 December 2011 Accepted: 31 October 2012 Published: 15 November 2012

\section{References}

1. Insall JN, Binazzi R, Soudry M, Mestriner LA: Total knee arthroplasty. Clin Orthop 1985, 192:13-22.

2. Bargren JH, Blaha JD, Freeman MAR: Alignment in total knee arthroplasty. Corr elated biomechanical and clinical observations. Clin Orthop 1983, 173:178-183.

3. Scuderi GR, Insall JN: The posterior stabilised knee prosthesis. Orthop Clin North Am 1989, 20:71-78.

4. Dennis DA, Channer M, Susman MH, Stringer EA: Intramedullary versus extramedullary tibial alignment systems in total knee arthroplasty. J Arthroplasty 1993, 8:43-47.

5. Bankes MJK, Back DL, Cannon SR, Briggs TWR: The effect of component malalignment on the clinical and radiological outcome of the Kinemax total knee replacement. Knee 2003, 10:55-60.

6. Coull R, Bankes MJK, Rossouw DJ: Evaluation of tibial component angles in 79 consecutive total knee arthroplasties. Knee 2003, 6:235-237.

7. Lewallen DG, Bryan RS, Peterson LF: Polycentric total knee arthroplasty. A ten-year follow-up study. J Bone Joint Surg Am 1984, 66:1211-1218.

8. Aglietti P, Buzzi R: Posteriorly stabilised total-condylar knee replacement Three to eight years' follow-up of 85 knees. J Bone Joint Surg Br 1988, 70:211-216.

9. Kuo CC, Bosque J, Meehan JP, Jamali AA: Computer-assisted navigation of total knee arthroplasty for osteoarthritis in a patient with severe posttraumatic femoral deformity. J Arthroplasty 2011, 26:976. e17-20.

10. Ishida K, Matsumoto T, Tsumura N, Kubo S, Kitagawa A, Chin T, Iguchi T, Kurosaka M, Kuroda R: Mid-term outcomes of computer-assisted total knee arthroplasty. Knee Surg Sports Traumatol Arthrosc 2011, 19:1107-1112.

11. Vanin N, Kenaway M, Panzica M, Jagodzinski M, Meller R, Krettek C, Hankemeier S: Accuracy of digital preoperative planning for total knee arthroplasty. Technol Health Care 2010, 18:335-340.

12. Nunley RM, Ellison BS, Zhu J, Ruh EL, Howell SM, Barrack RL: Do patientspecific guides improve coronal alignment in total knee arthroplasty? Clin Orthop Relat Res 2012, 470:895-902.

13. Seo JG, Moon YW, Park SH, Shim JW, Kim SM: An alternative method to create extramedullary references in total knee arthroplasty. Knee Surg Sports Traumatol Arthrosc 2012, 20:1339-1348.

14. Hasegawa M, Yoshida K, Wakabayashi H, Sudo A: Minimally invasive total knee arthroplasty: comparison of jig-based technique versus computer navigation for clinical and alignment outcome. Knee Surg Sports Traumatol Arthrosc 2011, 19:904-910.

15. Massai F, Conteduca F, Vadalà A, lorio R, Basiglini L, Ferretti A: Tibial stress fracture after computer-navigated total knee arthroplasty. J Orthop Traumatol 2010, 11:123-127.

16. Beldame J, Boisrenoult $P$, Beaufils P: Pin track induced fractures around computer-assisted TKA. Orthop Traumatol Surg Res 2010, 96:249-255.

\section{doi:10.1186/1758-2555-4-44}

Cite this article as: Mutsuzaki and Ikeda: Simple method for confirming tibial osteotomy during total knee arthroplasty. Sports Medicine, Arthroscopy, Rehabilitation, Therapy \& Technology 2012 4:44. 